\title{
Characterisation and compensation of magnetic distortions for the pixel Hybrid Photon Detectors of the $\mathrm{LHCb} \mathrm{RICH}$
}

\author{
G. Aglieri Rinella ${ }^{\mathrm{a}, \mathrm{b}}$, C. D'Ambrosio ${ }^{\mathrm{b}}$, R. Forty ${ }^{\mathrm{b}}, \mathrm{T} \cdot$ Gys $^{\mathrm{b}}$, M. Patel $^{\mathrm{b}}$, \\ D. Piedigrossi ${ }^{\mathrm{b}}$, A. Van Lysebetten ${ }^{\mathrm{b}, *}$ \\ ${ }^{a}$ Department of Electrical Engineering, V.le delle Scienze University of Palermo, Italy \\ ${ }^{\mathrm{b}}$ CERN, 1211 Geneva 23, Switzerland
}

Available online 1 September 2005

\begin{abstract}
The LHCb experiment at LHC, optimised for the study of CP violation, has two RICH detectors to provide particle identification in the momentum range $\sim 2-100 \mathrm{GeV} / c$. The stringent requirements on the photon detectors are met by the custom-made pixel Hybrid Photon Detector.

The photon detectors need to operate in the fringe field of the $\mathrm{LHCb}$ dipole magnet which will produce distortions of the image detected on the pixel chip which is encapsulated inside the HPD. This paper reports on the experimental characterisation of the image distortions caused by an external magnetic flux density. These measurements allow for the development of a parameterisation of the effects and a compensation algorithm, which are also presented.
\end{abstract}

(C) 2005 Elsevier B.V. All rights reserved.

PACS: $29.40 . \mathrm{Ka}$

Keywords: Hybrid Photon Detector; Magnetic distortions; S-distortion correction

\section{Introduction}

The LHCb experiment [1] at the LHC, dedicated to the study of $\mathrm{CP}$ violation, has two $\mathrm{RICH}$ detectors to provide particle identification over a wide momentum range $(\sim 2-100 \mathrm{GeV} / c)$. Three

\footnotetext{
*Corresponding author.

E-mail address: ann.van.lysebetten@cern.ch (A. Van Lysebetten).
}

different radiator materials are used (aerogel and the fluorocarbon gases $\mathrm{C}_{4} \mathrm{~F}_{10}$ and $\mathrm{CF}_{4}$ ). To detect the Cherenkov photons produced by charged particles in these materials with a high granularity $\left(2.5 \times 2.5 \mathrm{~mm}^{2}\right)$ over a large active area $\left(2.8 \mathrm{~m}^{2}\right)$, a high efficiency position sensitive single photon detector is needed. The adopted solution for the LHCb RICH detectors is the pixel Hybrid Photon Detector (HPD) [2]. The HPD (see Fig. 1) is a vacuum photon detector with a pixelated silicon 


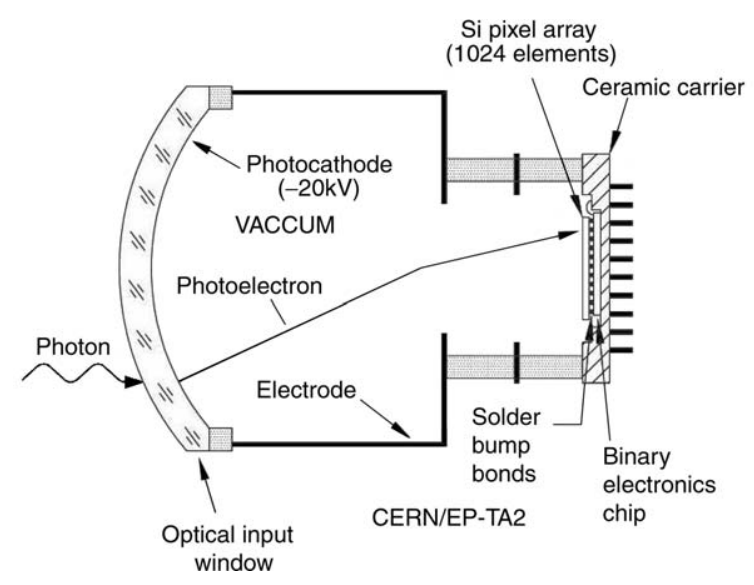

Fig. 1. A schematic of the HPD.

detector anode assembly. The tube has a quartz entrance window with a multialkali photocathode. Photoelectrons emitted from the photocathode are accelerated onto the anode assembly by a $20 \mathrm{kV}$ cross-focussing electron optics. The demagnification factor is 5 .

Both RICH detectors are situated in proximity of the $\mathrm{LHCb}$ dipole magnet, hence the 484 photon detectors need to operate in its fringe field. The HPD electron optics is sensitive to magnetic flux densities, in the same way as most image intensifiers [3]. The Lorentz force due to a field $\mathbf{B}_{\|}$parallel to the tube axis changes the electron trajectories causing them to rotate around the tube axis. This effect is visible as a significant $\mathrm{S}$ distortion and magnification of the anode image. A transverse magnetic flux density $\mathbf{B}_{\perp}$ with respect to the tube axis, causes a lateral shift of the electronic image.

Hence the HPDs are enclosed in primary magnetic shielding boxes designed to limit the field density flux to $2.5 \mathrm{mT}$ and $1.0 \mathrm{mT}$ in RICH1 and $\mathrm{RICH} 2$, respectively. The direction of the magnetic flux density at the RICH1 photon detector plane (mainly longitudinal) is different from the direction in RICH2 (mainly transverse) [4]. This is a direct consequence of the placement and orientation of the photon detector planes within the RICH detectors. The field value varies across the HPD plane, hence the field experienced by each HPD will be different. The magnetic flux

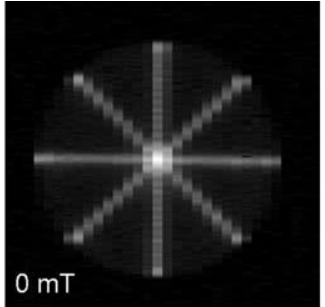

(a)

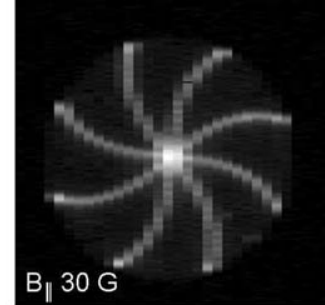

(b)

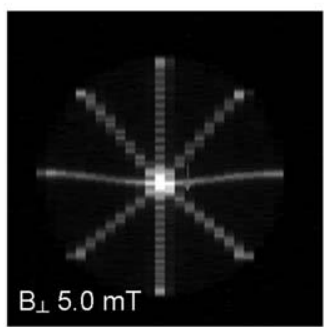

(c)

Fig. 2. (a) Image of the double cross with $B=0 \mathrm{mT}$. (b) Same light pattern with a $3.0 \mathrm{mT}$ axial field. (c) With a transverse field of $5.0 \mathrm{mT}$ applied.

density level inside the shielding boxes would still induce excessive distortions and even signal losses. Therefore, a local magnetic secondary shield of a high permeability alloy (MuMetal ${ }^{1}$ ) surrounds every HPD.

The results from detailed experimental characterisation are presented in this paper and are used in the development of a parameterisation procedure for distortion correction.

\section{The experimental set-up}

The measurements consisted of recording the photoelectron hit position on the anode when photons were shone on known locations on the photocathode while various magnetic flux densities were applied. The set-up used a DC LED to produce a double cross pattern (see Fig. 2(a)) and a digital projector for a programmable static light pattern (see Fig. 3(a)). The measurement of the spot centre and its error are determined from either a double gaussian fit or a weighted mean of the light intensity distribution. The magnetic flux

\footnotetext{
${ }^{1}$ Hereafter referred to as mumetal.
} 


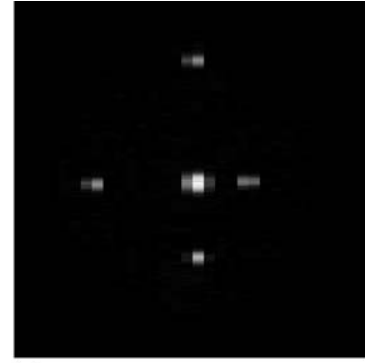

(a)

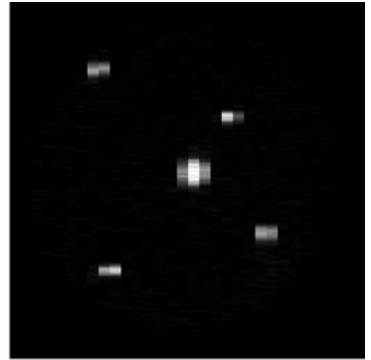

(b)

Fig. 3. (a) Test pattern taken without magnetic flux density. (b) The same pattern taken with a $5.0 \mathrm{mT}$ axial magnetic flux density.

densities were generated by a Helmholtz coil which could be rotated at different angles with respect to the HPD axis. Values up to $5.0 \mathrm{mT}$ could be obtained. Measurements with and without mumetal were done to characterise the limits and effectiveness of this local shield. The mumetal shield is a cylindrical tube of $140 \mathrm{~mm}$ length, $86 \mathrm{~mm}$ diameter, $0.9 \mathrm{~mm}$ thickness and protruding the HPD entrance window by $25 \mathrm{~mm}$. The insulation in the gap between the mumetal (at ground) and the HPD (at $-20 \mathrm{kV}$ ) is achieved with layers of Kapton ${ }^{2}$ foil.

\section{Characterisation and parametrisation of axial fields}

\subsection{Characterisation}

The distortion expected when a magnetic flux parallel to the HPD tube axis $\left(\mathbf{B}_{\|}\right)$is applied is a rotation and magnification of the image. The effect is non-uniform, as can be seen in Fig. 2(b). The rotation is due to the spiralling of electrons around the field direction, while they are accelerating from the photocathode towards the anode. With an unshielded HPD, a non recoverable signal loss is observed with $\left|\mathbf{B}_{\|}\right|>1.5 \mathrm{mT}$ : the cathode image is no longer fully contained within the anode area. Conversely, the image is fully contained within the anode even at the highest magnetic flux density

\footnotetext{
${ }^{2}$ Kapton trademarked polyimide film by DuPont.
}

Table 1

Maximum displacements of pattern points on the anode for various $\mathbf{B}_{\|}$field values measured with a shielded tube

\begin{tabular}{ll}
\hline $\mathbf{B}(\mathrm{mT})$ & $\begin{array}{l}\text { Maximum displacement } \\
\Delta d(\mathrm{~mm})\end{array}$ \\
\hline 1.0 & 0.9 \\
2.0 & 1.6 \\
3.0 & 2.3 \\
4.0 & 3.3 \\
5.0 & 4.3 \\
\hline
\end{tabular}

tested $(5.0 \mathrm{mT})$ when the HPD is shielded. The role of the mumetal shielding is therefore essential. At the maximum expected RICH1 field value of $3.0 \mathrm{mT}$, the distortions observed are bigger than the intrinsic resolution. ${ }^{3}$ This can be seen in Table 1, which displays the maximum displacements of the spot images between the reference pattern and the distorted pattern measured on the anode chip. This dictates the need to correct the HPD data for this distortion.

\subsection{Parameterisation}

The "demagnification law" is the function that relates the radial coordinate of the light spot at the HPD photocathode surface $r$ to the radial coordinate of focussed electrons at the anode plane $r^{\prime}$. It is well defined if the distortions have a rotational symmetry, as is the case for axial fields. Data points and the fitted functions are shown in Fig. 4 for the shielded tube, for field intensities ranging from $0.0 \mathrm{mT}$ to $5.0 \mathrm{mT}$. The data points are well fitted with a polynomial relation with coefficients $a_{i}$ depending on the field value

$$
\begin{aligned}
& r^{\prime}=a_{1}(B) r+a_{2}(B) r^{2} \\
& a_{i}(B)=\sum_{j \leqslant 3} a_{i, j} B^{j},
\end{aligned}
$$

with $B$ defined as the axial component $\mathbf{B}_{\|}$of the field applied and $B^{j}$ signifies $\mathbf{B}_{\|}$to the power $j$. Also in the case of the rotation law (relating the rotation angle to the radial coordinate of the light spot $r$ ) a polynomial fit to the data

\footnotetext{
${ }^{3}$ The HPD pixel size is $0.5 \times 0.5 \mathrm{~mm}^{2}$ in $\mathrm{LHCb}$ readout mode.
} 


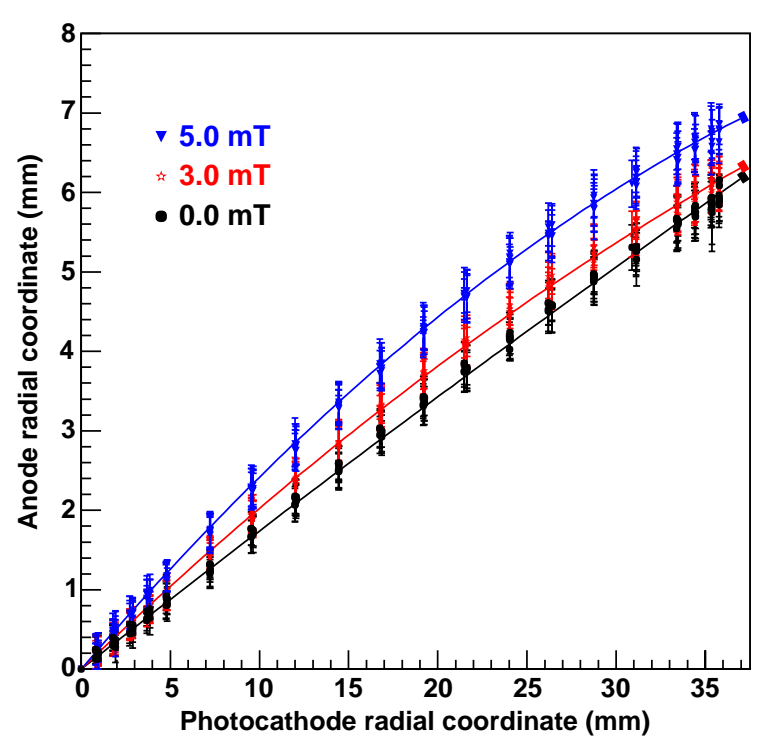

Fig. 4. Demagnification law: radial coordinate of focussed electrons at the anode plane versus radial coordinate of the light spot at the photocathode plane for increasing axial magnetic flux density.

can be assumed:

$\Delta \phi=a_{3}(B)+a_{4}(B) r^{2}+a_{5}(B) r^{3}$

$a_{i}(B)=\sum_{j \leqslant 3} a_{i, j} B^{j}$

The data points and the result of the fit is shown in Fig. 5. The coefficients are functions of the field applied and were obtained from a best fit to the set of experimental data. Both laws can be used to reconstruct the photon position from the pixel hit under assumption that the value of the applied $\mathbf{B}_{\|}$ field is known. The algorithm was tested on the double cross patterns. The photon position was reconstructed from these recorded data and the average reconstruction bias (defined as the difference between the true position and the reconstructed coordinate) was evaluated (see Table 2) for different values of the $\mathbf{B}$ field applied. These bias errors should be compared to the spatial resolution of the HPD due to the pixel size which is $2.5 \mathrm{~mm} / \sqrt{12} \cong 0.72 \mathrm{~mm}$. Uncertainties on the coordinates of the centres in the cathode and anode plane, used to reconstruct radial distances

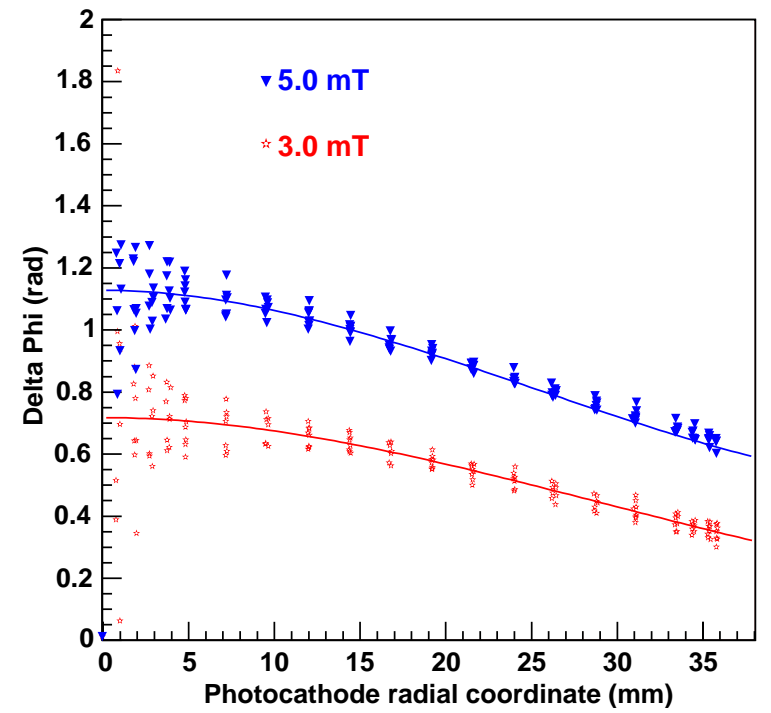

Fig. 5. Rotation laws: angle of rotation of the imaged spots on the anode plane versus photocathode radial coordinate of the light spot for increasing axial magnetic flux density.

Table 2

Photon position reconstruction bias measured on HPD entrance window

\begin{tabular}{ll}
\hline B $(\mathrm{mT})$ & Average bias $(\mathrm{mm})$ \\
\hline 0.0 & 0.82 \\
1.0 & 1.24 \\
2.0 & 1.40 \\
3.0 & 1.78 \\
\hline
\end{tabular}

and the rotation angle, are believed to give a large contribution to the total reconstruction bias error.

The model could also be used to estimate the value of the applied $\mathbf{B}_{\|}$field. The procedure is based on the fitting of the data taken with a test pattern in absence and presence of the $\mathbf{B}_{\|}$field to be determined. The test pattern consists of 5 light spots, a larger central one and a set of smaller ones at increasing radii from the central one (see Fig. 3). The pattern is kept simple to simplify the correspondence between patterns with and without applied field. The results of the method applied to different test runs taken with various magnetic flux densities are given in Table 3 . The $\mathbf{B}_{\|}$ estimation precision is better than $0.3 \mathrm{mT}$. 
Table 3

Axial field estimator error

\begin{tabular}{ll}
\hline $\mathbf{B}_{\|}(\mathrm{mT})$ & Estimated field $(\mathrm{mT})$ \\
\hline 1.0 & $0.90 \pm 0.20$ \\
2.0 & $2.05 \pm 0.27$ \\
3.0 & $3.24 \pm 0.27$ \\
4.0 & $4.08 \pm 0.24$ \\
5.0 & $5.07 \pm 0.19$ \\
\hline
\end{tabular}

\section{Characterisation of transverse field effects}

The effect of a transverse field is a non uniform translation of the image perpendicular to the direction of the applied magnetic flux density $\mathbf{B}_{\perp}$ (see Fig. 2(c)). The mumetal protection in the transverse case is much more efficient than for the axial case; the distortion effects reduce by a factor $\sim 20$. The distortions observed at $5.0 \mathrm{mT}$ are of the order of the intrinsic resolution of the system and, up to $3.0 \mathrm{mT}$, no significant distortions are observed. These results are shown in Table 4 and indicate that there may be no need for a distortion correction of the transverse component in $\mathrm{RICH} 2$.

\section{Effects from reduced $\mathrm{HV}$ and remnant magnetisation}

The influence of operation at reduced cathode high voltage on the magnetic distortions was investigated with the shielded tube. An axial field of $3.0 \mathrm{mT}$ was applied. Some differences from $-20 \mathrm{kV}$ start to be visible at cathode voltages of $-16 \mathrm{kV}$; a somewhat larger rotation angle is observed $(0.77 \mathrm{rad}$ instead of $0.72 \mathrm{rad}$ at $-20 \mathrm{kV})$. No differences in distortions were observed for the transverse configuration.

The HPD electrodes and the mumetal shield are made of magnetic materials, hence even in absence of an external magnetic flux density, a remnant flux density may be present and cause distortions. Although significant effects (a global offset of $1.2 \mathrm{~mm}$ of the photocathode image on the anode) were observed in the transverse configuration for
Table 4

Maximum displacements of pattern points on the anode for various $\mathbf{B}_{\perp}$ field values

\begin{tabular}{ll}
\hline $\mathbf{B}_{\perp}(\mathrm{mT})$ & $\begin{array}{l}\text { Maximum displacement } \\
\Delta d(\mathrm{~mm})\end{array}$ \\
\hline 3.0 & 0.45 \\
5.0 & 0.73 \\
\hline
\end{tabular}

the unshielded tube, negligible effects were seen for the shielded tube (offset of less than a quarter of the $\mathrm{LHCb}$ pixel size). No effects related to remnant field were observed in the axial case.

\section{Conclusions}

The HPD developed to fulfill the RICH detector requirements of the $\mathrm{LHCb}$ experiment will need to operate in the fringe field of the $\mathrm{LHCb}$ dipole magnet. A detailed experimental characterisation indicated the necessity of mumetal local shielding and proved that HPDs equipped with this shielding are operational in fields of $5.0 \mathrm{mT}$ (more than double the expected value). A distortion correction algorithm was developed for axial fields and was used successfully for testing the photon position reconstruction and for the estimation of the axial magnetic flux density component. The measurements indicated negligible distortions caused by transverse fields and hence no distortion corrections are expected to be necessary for the transverse component in the expected $\mathbf{B}$ field in $\mathrm{RICH} 2$.

\section{References}

[1] LHCb collaboration, LHCb reoptimized Detector design and Performance Technical Design Report, CERN/LHCC 2003-030, 2003.

[2] N. Kanaya, Performance study of the pixel hybrid photon detectors for $\mathrm{LHCb} \mathrm{RICH}$, these proceedings.

[3] E. Gronenschild, Med. Phys. 26 (1999) 12.

[4] M. Patel, et al., Magnetic shielding studies of the LHCb $\mathrm{RICH}$ photon detectors, these proceedings. 